\title{
Imaging of concentration distributions and hydrogen evolution on corroding magnesium exposed to aqueous environments using Scanning Electrochemical Microscopy
}

Javier Izquierdo, ${ }^{\mathrm{a}, \mathrm{b}}$ Bibiana M. Fernández-Pérez, ${ }^{\mathrm{a}}$ Dániel Filotás, ${ }^{\mathrm{c}}$ Zsuzsanna Öri, ${ }^{\mathrm{c}}$ András Kiss, ${ }^{\mathrm{c}}$ Romen T. Martín-Gómez, ${ }^{\mathrm{a}}$ Lívia Nagy, ${ }^{\mathrm{d}}$ Géza Nagy, ${ }^{\mathrm{c}}$ Ricardo M. Souto ${ }^{\mathrm{a}, \mathrm{b}}$

a Department of Chemistry, Universidad de La Laguna, P.O. Box 456, E-38200 La Laguna, Tenerife, Canary Islands, Spain.

b Institute of Material Science and Nanotechnology, Universidad de La Laguna, E-38200 La Laguna (Tenerife), Spain.

c Department of General and Physical Chemistry, Faculty of Sciences, University of Pécs, Ifjúság útja 6, 7624 Pécs, Hungary.

d János Szentágothai Research Center, University of Pécs, Ifjúság u.20. Pécs, 7624 Hungary.

\begin{abstract}
Scanning Electrochemical Microscopy (SECM) is presented as an essential tool for the local characterization of the still uncertain mechanism for magnesium corrosion. The reaction leading to magnesium release and hydrogen evolution from separated magnesium cathodes and anodes has been imaged using an adequate combination of the operation modes available in SECM. Magnesium ion selective microelectrodes (Mg-ISME's) were used for the visualization of the heterogeneously distributed release of magnesium (II) species. Antimony microelectrodes detected the $\mathrm{pH}$ gradients in the adjacent electrolyte resulting from either water or magnesium electrolysis, whereas platinum microdiscs were used to monitor the concomitant local evolution of hydrogen. Alkalization and $\mathrm{H}_{2}$ generation were observed over the magnesium strip polarized as cathode, whereas a small local acidification was observed above the strip polarized anodically, at which extensive heterogeneous magnesium release was also imaged.
\end{abstract}

Keywords: Magnesium; SECM; hydrogen evolution; pH gradients; microelectrode probe. 


\section{Introduction}

Magnesium and its alloys have a great potential for several industrial and biomedical applications due to their non-toxicity and high strength-weight ratio, though their practical use is severely limited by their poor corrosion resistance in most aqueous environments [1-4]. In order to slow down this degradation, it is mandatory to gather precise knowledge about the mechanism of the process. Unfortunately, the mechanism of magnesium corrosion remains controversial, and it presents certain unique features when compared to the corrosion of other active metals, in particular with regard to the involved hydrogen evolution. While the main cathodic half-reaction leads to proton reduction into hydrogen gas, this reaction has been recorded to occur on magnesium not only under cathodic polarization, as expected, but also when increasing anodic overpotentials are applied to the material. This phenomenon, usually termed as the "Negative Difference Effect" [1,3], has been traditionally invoked to support the formation of magnesium (I) cations as intermediate species during the degradation of this metal [1]. That is, oxidation of magnesium at the anodic sites would produce $\mathrm{Mg}(\mathrm{I})$ species which subsequently react with water producing the hydrogen gas [1,5]. Indeed, there are published reports suggesting that the apparent valence of the anodic half-reaction during the corrosion of magnesium was smaller than 2.0, which has been regarded the main evidence of the mechanism involving univalent $\mathrm{Mg}$ cations [6]. However, the advent of a new range of sensitive microelectrochemical and spectroelectrochemical methods are delivering new experimental evidences that anodic dissolution produces solely $\mathrm{Mg}(\mathrm{II})$ instead, through a 2-electron exchange process [7-11]. Coupling of electrochemical measurements with atomic emission spectroelectrochemistry supported that the apparent unbalance between $\mathrm{Mg}$ dissolution and hydrogen release rather arises from the formation of both soluble and insoluble $\mathrm{Mg}^{2+}$ species, the latter increasing with the elapse of time, thus effectively correlating to an $n=2$ mechanism [12]. This would relate to the formation of dark bi-layered film precipitates on anodically polarised magnesium in chloride solutions [13-16], because this film has been reported to exhibit enhanced catalytic activity towards hydrogen evolution [15,17]. These latter observations can be regarded more consistent with the expectation that no direct determination of the $\mathrm{Mg}(\mathrm{I})$ should be feasible due to its very likely instability in aqueous solution [18], and some of the processes involved in the experiments traditionally considered to indirectly prove the existence of $\mathrm{Mg}(\mathrm{I})$ some decades ago [19] may have been possibly misunderstood [20]. Therefore, the actual sites of hydrogen evolution from corroding magnesium remains uncertain [21,22], and it has become the most critical aspect to be unveiled for the actual corrosion mechanism to be established, in order to gain effective control on the corrosion process for the application of magnesium-based materials in practical situations.

The electrochemical reactions occurring in metal corrosion originate from the occurrence of microcathodes and microanodes over the surface undergoing degradation in spite of apparent homogeneity. Regarding the corrosion of magnesium, the process is highly heterogeneous, and leads to the formation of $\mathrm{pH}$ gradients in the adjacent electrolyte and the release of hydrogen bubbles from water hydrolysis. In this context, several efforts have recently been performed to understand the heterogeneous aspects of the corrosion of magnesium and magnesium-based materials and its convergence with the macroscopic apparently-homogeneous observations, in order to adequately elucidate the actual mechanism. The work involved the determination of the spatially distributed anodes and cathodes on the metallic surfaces using the scanning vibrating electrode technique (SVET) $[7,11]$ and the scanning reference electrode technique [23]; or the local detection of magnesium concentration and $\mathrm{pH}$ profiles using either the scanning ion electrode technique (SIET) [24] or the potentiometric mode of the scanning electrochemical microscope (SECM) [10].

SECM is a microelectrochemical technique that results particularly attractive because it combines high spatial and electrochemical resolutions, thus constituting a very promising technique for the characterization of heterogeneously distributed electrochemical processes like those occurring on a corroding surface, and in particular to investigate the mechanism of magnesium corrosion. Yet, amperometric SECM operation has been scarcely employed due to the difficult selection of a suitable redox mediator for imaging [25]. Firstly, the corrosion product (i.e., the magnesium ions), cannot be employed because its redox conversion occurs beyond the range of stability of water. Neither dissolved 
oxygen is an option because this molecule plays a minor role in the corrosion of magnesium, thus no significant consumption occurs on the corroding sample. Furthermore, evolving hydrogen is a strong reducing agent that makes impractical the addition of redox mediators in their oxidized form, whereas the potential range of the hydrogen oxidation reaction (HOR) may occur for $E>-0.7 \mathrm{~V}$ vs. Ag/AgCl [26,27], strongly dependent on $\mathrm{H}_{2}$ pressure and ultimately on $\mathrm{pH}$. As result, feedback modes have only served to characterize the uniformity of surface treatments on magnesium by using Fe(II)-containing redox mediators [27-29].

Greater success was observed by using ion selective microelectrodes (ISME) as probes, allowing the concentration distribution of dissolving $\mathrm{Mg}^{2+}$ ions to be imaged with high spatial resolution $[10,30,31]$. The combination of the above mentioned methodologies with the analysis of $\mathrm{pH}$ gradients in the vicinity of active corroding surfaces, making use of iridium [27] or antimony-based [10,31] dual amperometric/potentiometric probes, has been presented as essential information sources for the analysis of the evolution of the species involved in the corrosion process. Recently, an additional promising route for SECM characterization of magnesium corrosion has been proposed in two separate studies [27,32]. They have demonstrated that chemical imaging can be performed using the oxidation reaction of the $\mathrm{H}_{2}$ (HOR) evolving from corroding magnesium surfaces. SECM images of corroding $\mathrm{Mg}$ alloys allowed to investigate time evolution and the effect of active site sizes. The SECM operation is actually a sample generation-tip collection mode, and its applicability for the determination of the corrosion rate of magnesium is based on the assumption that molecular hydrogen is produced in equimolar amounts to dissolved magnesium [27,32]. That is, hydrogen evolution is regarded to be a purely cathodic process [33]. But other recent reports state that hydrogen evolution occurs over anodically-polarized surfaces too (i.e., the Negative Difference Effect) [5,6,16,21]. Therefore, it is of paramount importance to determine the source of hydrogen for the unambiguous interpretation of SECM measurements using the HOR, as well as to establish the complete corrosion mechanism for this metal.

This contribution reports an SECM investigation concerning the source of hydrogen evolution occurring over magnesium surfaces undergoing corrosion. Cathodic and anodic magnesium activities have been separated and controlled using an Mg-Mg pair interconnected through a small circuit containing a battery and combinations of resistances, in order to precisely control the total current flowing through the samples. In this way, spatial distributions of evolving hydrogen and $\mathrm{pH}$ gradients were monitored under various externally-imposed electrical polarizations to the metal, and the subsequent release of magnesium cations from the metallic surfaces, as measured with the Mg-ISME under equivalent conditions.

\section{Experimental}

\subsection{Reagents and samples}

Magnesium metal ribbon of 99\% purity supplied by Panreac (Barcelona, Spain), with a cross section of $200 \mu \mathrm{m} \times 3 \mathrm{~mm}$, was employed as substrate material. According to the manufacturer, impurities consisted of 0.005 wt.\% Cu, 0.05 wt.\% Fe, 0.005 wt.\% Ni, 0.005 wt.\% Pb, and 0.02 wt.\% Zn. Two equal metal strips were fixed vertically in a two-compound resin (Epofix Kit, Struers, Ballerup, Denmark), facing up their rectangle cross sections as the active surfaces to be analyzed, and separated by ca. 7-8 mm. The resulting mount with the embedded metal strips, with circular surface of $3 \mathrm{~cm}$ diameter, was flat ground with $\mathrm{SiC}$ paper and finished using $0.3 \mu \mathrm{m}$ particle size $\mathrm{Al}_{2} \mathrm{O}_{3}$ ethanolic suspension, rinsed with ethanol and dried in air. It was finally wrapped around laterally by Sellotape to form a small container filled with approximately $4 \mathrm{~mL}$ of $1 \mathrm{mM} \mathrm{NaCl}$ as test electrolyte. Analytical grade sodium chloride from Merck (Darmstadt, Germany) was used to prepare this solution. The same supplier provided the magnesium chloride hexahydrate for the preparation of the standard solutions employed for the calibration of the magnesium selective microelectrodes. Every solution was prepared using ultrapure deionised water (18.0 $\mathrm{M} \Omega \mathrm{cm}$, Millipore, Billerica, MA, USA). 


\subsection{Scanning electrochemical microscopy measurements}

Measurements were performed with an SECM equipment built by Sensolytics (Bochum, Germany), controlled with personal computer. The electrochemical interface was a PalmSens bipotentiostat (Utrecht, The Netherlands). Video camera assistance was employed to place the sensing probe in close proximity to the substrate. In addition, if amperometric determination was possible, i.e., when either Pt or Sb-based ultramicroelectrodes (UME's) were in operation, a more precise determination of the surface location was attained by recording $z$-approach curves over a site on the resin mould equidistant from both magnesium strips. The negative feedback function measured for the oxygen reduction current was employed in the case of the Pt microelectrode set at $-0.65 \mathrm{~V}$, whereas positioning of the antimony electrode was performed using the alternating current (AC-SECM) mode [34] to avoid electrochemical modification of the antimony oxide film. An AC potential signal of $10 \mathrm{mV}_{\mathrm{pp}}$ amplitude and $100 \mathrm{kHz}$ frequency was then applied to the open circuit potential of the antimony electrode. Once the substrate surface was located and the tip height adjusted to ca. $20 \mu \mathrm{m}$, SECM data were recorded by moving the probe parallel to the surface either across the shortest $200 \mu \mathrm{m}$ cross section of the magnesium strip or along the whole area where the metal was located. A compromise situation for the scan rate was established for each electrode probe in order to image the metal surfaces fast enough before they were dramatically altered due to the corrosion process, but still slow enough to obtain reproducible and laterally-resolved images.

All the amperometric and potentiometric SECM probes employed, i.e. Pt and Sb-based UME's, and the Mg-ion selective microelectrode (Mg-ISME), were fabricated following well established methods, and used as working electrodes. The electrochemical cell was completed with an $\mathrm{Ag} / \mathrm{AgCl} / \mathrm{KCl}(3 \mathrm{M})$ electrode as reference and a Pt wire as counter electrode when amperometric operation was required. All potential values in this work are referred to this reference electrode.

Platinum microelectrodes were fabricated from $12.5 \mu \mathrm{m}$ diameter platinum wire $(99.9 \%$ purity, Goodfellow, Cambridge, UK) following a protocol described in [35]. In brief, 2-3 cm length Pt wires were inserted into boron silicate capillaries of $1.125 \mathrm{~mm}$ and $1.5 \mathrm{~mm}$ inner and outer diameter, respectively (Hilgenberg GmbH, Masfeld, Germany). Then, one end of the capillary was heated up so that 2-3 mm of one end of the Pt wire could be sealed into the glass. The electric connection between the protruding non-sealed Pt wire inside the capillary and the measuring device was ensured with silver conductive epoxy (RS Components Ltd., Oxford, UK) and a copper wire. The final assembly was polished with $\mathrm{SiC}$ paper in order to expose a $12.5 \mu \mathrm{m}$ diameter Pt microdisc surrounded by an insulating glass surface, and finally with $0.3 \mu \mathrm{m} \mathrm{Al}_{2} \mathrm{O}_{3}$ aqueous suspension.

Antimony microdisc electrodes of 30-35 $\mu \mathrm{m}$ diameters were prepared using high purity antimony in powder presentation (Aldrich, Saint Louis, MO, USA) as described and sketched in [10,36]. Prior to the final assembly of the electrodes, antimony was melted under flame and inserted by suction into a Pyrex glass capillary, which was thereafter pulled under heating. The resulting pulled Sb-containing capillaries were inserted and glued inside the same boron silicate capillaries employed in the fabrication of the platinum electrodes. In this case, the electric contact between the antimony and the copper wires used for the connection to the measuring device was achieved using a small quantity of liquid mercury. For the calibration of the antimony electrode, $\mathrm{pH}$ buffer standard solutions in the 4-11 range (VWR International Eurolab S.L., Barcelona, Spain) were used.

The Mg-ISME's were fabricated in the configuration of a solid-contact ion selective membrane microelectrode as explained and sketched in [31]. Bis-N,N-dychlohexyl-malonamide selective ionophore was synthesized by procedure given in [37]. For the preparation of the cocktail, selectophore grade high-molecular weight polyvinyl chloride (PVC), ortho-nitrophenyl octyl ether (o-NPOE), potassium tetrakis(4-chlorophenyl)-borate (PTCB), and tetrahydrofurane (THF) as liquid solvent were purchased from Fluka (Buchs, Switzerland). They were mixed with the ionophore in the ratios given in Table 1, and the final cocktail was introduced under suction in the boron silicate capillaries, once pulled under heat at one end. For the solid contact between the cocktail and the contact pad to the measuring equipment, carbon fibres of $33 \mu \mathrm{m}$ diameter, provided by Specialty Materials (Lowell, MA, USA) as a generous gift, were used. The conductivity of the fibres was enhanced by electrochemical polymerization of 3,4-ethylenedioxythiophene (EDOT) monomer obtained from Starck (Golar, 
Germany), in 1-butyl-3-methyl-imidazoliumhexafluorophosphate (BMIM $\left.{ }^{+} \mathrm{PF}_{6}{ }^{-}\right)$ionic liquid solvent from Solvent Innovation (Cologne, Germany), and fibres were finally sealed with the copper wire for the electrical connection. Finally, the ion selective electrodes were calibrated in $\mathrm{MgCl}_{2}$ solutions within the $10^{-6}$ to $10^{-1} \mathrm{Mg}^{2+}$-concentration range with $1 \mathrm{mM} \mathrm{NaCl}$ as supporting electrolyte. Activity coefficients were estimated using the Debye-Hückel approach.

\subsection{Experimental procedures}

Hydrogen gas evolution was monitored with the Pt UME set at $0 \mathrm{~V}$ (i.e., quasi-diffusional conditions [26,27,32]), whereas $\mathrm{pH}$ and $\mathrm{Mg}(\mathrm{II})$ concentration gradients were determined in the potentiometric mode using the $\mathrm{Sb}$ and the $\mathrm{Mg}$ ion selective microelectrodes, respectively, $[10,30,31,36]$. The electric condition of the magnesium strips was controlled by connecting the electric circuit depicted in Figure 1 at the rear of the mould. The circuit contains a $1.5 \mathrm{~V}$ battery and two resistances, allowing different $R_{\mathrm{s}}-R_{\mathrm{p}}$ combinations to be tested within the $0-40 \mathrm{k} \Omega$ range, as well as a digital ammeter connected in series in order to monitor the actual current flowing through the two magnesium surfaces exposed to the electrolyte. In this way, anodic and cathodic activities were separated between the metal strips, and different extents of the potential difference imposed between them could be produced. $R_{\mathrm{s}}-R_{\mathrm{p}}$ combinations were chosen to produce a sequence of increasing corrosive conditions for the anodically active surface, as characterized by the global current measured at the ammeter. Freshly prepared magnesium surfaces were used in every case, so after applying either a given polarization conditioning to the magnesium strips, or using a certain SECM probe for chemically-resolved imaging, the specimen was abraded and polished again before imaging the other strip, or following replacement of the sensing microelectrode probe to employ a different SECM operation mode.

\section{Results}

The scanning electrochemical microscope (SECM) employs a microelectrode as sensing probe (tip) that is rastered in close proximity to a solid surface to spatially-resolve any chemical heterogeneities and surface reactivity distributions. This is achieved by recording an electrochemical signal at the microelectrode that correlates with chemical species present at the interface or differences in surface conductivity. Three main approaches can be taken in order to detect and monitor at the probe an electrochemical signal directly related to the chemical characteristics of the investigated substrate, namely amperometric, potentiometric and AC operation modes. Amperometric operation of the SECM involves monitoring an electrochemical current with the microelectrode tip, typically built using a noble metal such as platinum. This faradaic response is originated by the electrochemical reaction of an electroactive species either placed in solution or evolving from the substrate.

When the tip current monitors the flux of an electroactive species released from the material under investigation, the SECM is said to be operated in the substrate generation-tip collection mode (SG/TC), which is useful to detect active sites on a surface. Due to the relative sizes of the substrate and the tip, the collection efficiency in mode SG/TC is very low, and greatly varies with the geometry of the microelectrode and its active material. Therefore, the method cannot be employed for quantification of the total amount of species released from a reacting surface, but local differences in reactivity (i.e., spatial distribution of a certain chemical species) can be observed by comparing the different currents measured at the tip while closely rastering the surface under investigation. The suitability of this operation mode for the investigation of hydrogen evolution from a corroding surface was already demonstrated by Fushimi et al. in the case of polarized Fe-3at.\%Si alloy in HCl solution [26], and it has been recently employed for the characterization of corroding magnesium too [27,32]. In this case, the Pt UME tip is polarised at $0 \mathrm{~V}$ in order to oxidize hydrogen released from the reactive substrate, thus allowing image the distribution of sites with hydrogen gas evolution (see Figure 2A). In order to increase the collection efficiency as much as possible in this work, the $12.5 \mu \mathrm{m}$ diameter Pt microdisc was surrounded by an almost 100 times larger insulating glass surface, as to produce a sufficiently 
large confined volume of electrolyte between the substrate and the tip for the detection of the chemical species evolving from the surface. In this way, a higher collection of evolving species from the active surface was attained, though at the cost of disturbances caused by the movement of the hydrogen bubbles formed at the magnesium portion just below the tip. In addition, precise positioning of the tip electrode with respect to the investigated surface is also required in this operation mode, as to satisfactorily resolve the conflicting requirements of higher resolution and greater collection efficiency provided by the operation at small tip-substrate distances, with the possible interference of reaction products of the redox conversion occurring at the tip towards the corrosion process of the investigated metal. As shown in the sketch given in Figure 2A, the reaction product of hydrogen oxidation at the tip produces hydrogen ions, which effectively affect the corrosion rate of magnesium. In order to minimize the interference of $\mathrm{H}^{+}$ions while recording the release of hydrogen gas with sufficient resolution, a constant tip-substrate distance equal to 1.5 tip diameters was selected. Precise determination of the tip-substrate distance was achieved by recording $z$-approach curves for the oxygen reduction reaction on locations of the resin at either side of the magnesium strips [38] (see diagram in Figure 2D). Once the tip-substrate distance was determined from the $z$-approach curve, the tip was removed to the chosen working distance. Then, an average baseline for the faradaic current related to hydrogen oxidation at the tip was determined while rastering over the resin sufficiently far from the metal strips, since hydrogen can only originate from the corroding metal surface. Any current bursts measured at the tip would then correlate to the amount of hydrogen released from the magnesium strips at different sites for each test condition described below.

Water electrolysis on corroding magnesium produces $\mathrm{OH}^{-}$ions in addition to hydrogen gas evolution as shown in Figure 2B. As result, local alkalization of the electrolyte occurs in the proximity of the sites for the hydrogen evolution reaction (HER). On the other hand, soluble magnesium ionic species may undergo hydrolysis, and local acidification would then occur [39]. Therefore, $\mathrm{pH}$ distributions in the solution volume adjacent to the corroding metal surface should occur, and can be imaged using the potentiometric mode of the SECM by using a pH-sensitive probe [36]. The potential response of antimony oxides to $\mathrm{pH}$ was employed for monitoring the local $\mathrm{pH}$ distributions as SECM probe. For the positioning of the potentiometric probe with respect to the investigated surface, operation of the SECM in the alternating current mode was employed [34] to avoid faradaic reactions to occur at the sensing probe because it will modify the composition of the $\mathrm{Sb} / \mathrm{Sb}_{2} \mathrm{O}_{3}$ microelectrode. In this arrangement, the response of the instrument is sensitive to variations in the local conductivity related to the length of electrolyte comprised between the tip and the substrate, as indicated in the sketch given in Figure 2E.

Finally, potentiometric operation mode of SECM using an ion selective microelectrode (ISME) as probe was also employed. As it can be observed in Figure 2C, precise and selective detection of an ionic species released into the solution could thus be monitored [40]. This principle was employed to quantify the release of magnesium(II) species as shown in Figure 2C.

Ion selective potentiometric probes were calibrated using standard $\mathrm{pH}$ and $\mathrm{MgCl}_{2}$ solutions, and the calibration curves are shown in Figure 3. Linear relationships were found with slopes amounting -40.43 and $-24.78 \mathrm{mV}$ decade $^{-1}$ for Sb-based and Mg-ion selective microelectrodes, respectively. A sub-Nernstian type response was obtained for the $\mathrm{pH}$ microsensor, instead of the -59 $\mathrm{mV}$ decade $^{-1}$ theoretical value, though this result is not uncommon for the $\mathrm{Sb} / \mathrm{Sb}_{2} \mathrm{O}_{3}$ surfaces $[10,36]$. The red dots in either of the graphs were not considered for the establishment of the linear correlation, as linearity was not maintained at those concentration limits. Not to avail, strongly acidic or alkaline media may induce corrosive attack or critical surface modifications in the antimony surface, and micromolar magnesium concentration range is already below the detection limit.

SECM was first employed to characterize the behaviour of a freely-corroding magnesium sample in the chloride-containing solution. That is, the magnesium strips contained in the sample were left without any electric connection at the rear of the mould, thus effectively remaining unpolarized during the measurements. Amperometric detection of hydrogen as well as monitoring of the $\mathrm{pH}$ evolution occurring over the freely-corroding magnesium surfaces were thus recorded using the $\mathrm{Pt}$ and the $\mathrm{Sb} / \mathrm{Sb}_{2} \mathrm{O}_{3} \mathrm{UME}$ 's as probes, respectively. Under these conditions, alkalization of the surrounding environment is expected [10], which we herein aimed to correlate with the evolution of hydrogen gas 
from the corroding metal. Typical results of these features are shown in Figure 4. Figure 4A evidences detection of hydrogen gas generated at the magnesium surface as result of the corrosion process, and the tip currents related to the process show a maximum value reaching $115 \mathrm{pA}$. Hydrogen evolution is indeed accompanied by the alkalization of the adjacent electrolyte due to the cathodic half-reaction of the corrosion process (cf. Figure 4B), reaching pH values up to 11.5, in good agreement with previous observations [10]. Yet, these measurements do not allow the cathodic and anodic sites on the corroding surface to be separated, neither to establish the half-cell reaction involved in the distributed release of hydrogen gas monitored in these graphs.

Separation of the anodic and cathodic half-cell reactions between two identical magnesium strips was accomplished through their electrical interconnection using the circuit drawn in Figure 1 with combinations of 0,20 and $40 \mathrm{k} \Omega$ resistances included as $R_{\mathrm{s}}$ and $R_{\mathrm{p}}$ in the external circuit. In that way, the bigger the parallel resistance and the smaller the series one, the more current will flow through the circuit in series (i.e., through the magnesium surfaces). On the other hand, if the parallel resistance is made smaller while maintaining a high resistance in series, the electric current will preferably flow through the branch in parallel, thus avoiding the passage through the ammeter and the magnesium strips. In this arrangement, the application of an external forced corrosion to the magnesium strips was prevented in the system.

Figure 5 depicts selected amperometric and potentiometric SECM scans recorded over the two magnesium surfaces subjected to polarization by forcing a current to flow between the two strips through the electronic circuit placed at the rear of the sample. In this way, one of the strips behaves as the anode of the electrolytic cell, and the other as the cathode, and different currents are driven through them by conveniently adjusting the resistors in the external circuit. The biggest spatial concentration distributions in the system were observed when the highest current of $180 \mu \mathrm{A}$ was recorded at the ammeter. Vigorous hydrogen evolution occurred above the magnesium strip behaving as the cathode, which was amperometrically oxidized at the Pt UME giving a peak tip current amounting $0.58 \mathrm{nA}$ (cf. Figure 5A). Though this faradaic current does not provide a measure of the total hydrogen evolving in the system due to the small collection efficiency of the SG-TC configuration of the SECM, it can be employed to compare the different extent of the HER when the electrical conditions imposed to the system are varied between the experiments. Next, a strong alkalization was observed above the magnesium strip operating as cathode, with potential responses of the antimony tip beyond its potentiometric response range (apparently higher than $\mathrm{pH}=13.5$ in Figure 5C). These trends are still visible in the other less aggressive conditions shown in the same figure. In fact, the results recorded over the magnesium cathode for the other two polarization conditions shown, corresponding to $35 \mu \mathrm{A}$ and $110 \mu \mathrm{A}$ global currents flowing through the two magnesium surfaces, resemble quite closely the situation previously described in Figure 4 for an unpolarized magnesium surface, i.e., under freely corroding conditions. Nevertheless, alkalization in this case is more extended than in the spontaneous case, reaching even beyond the calibration range for the sensing probe, an indication of enhanced hydrogen gas formation on the mildly polarized magnesium cathode compared to its behaviour under freely corroding conditions.

Conversely, the signals related to $\mathrm{pH}$ gradients and to hydrogen oxidation are small and very noisy over the magnesium strip polarized as the anode of the microelectrochemical cell, especially for the smaller currents forced in the system by using the arrangement of resistors in the external electric circuit. Yet, these results indicate hydrogen evolution. The noisy signals are related to agitation effects in the electrolyte due to the formation and detachment of hydrogen bubbles, which could be observed with the videocamera during the experiment. It must be noticed that, even if they move almost exclusively in an upward motion, electrolyte displacement occurs in the thin electrolyte cell [25]. This effect is more notorious for the smaller current scale and smaller signals recorded over the anodic magnesium strip as compared to the cathodic one (cf. Figures 5B and 5A, respectively). Indeed, the only noise-free peak for the oxidation current of hydrogen oxidation at the Pt tip in Figure 5B occurred for the less aggressive corrosive conditioning, corresponding to the passage of $35 \mu \mathrm{A}$ total current between the metal strips (see black line in Figure 5B), when hydrogen evolution should be less vigorous. More noisy signals are found for the scan lines measured over the anodic magnesium strip in the case of higher current passage through the circuit, yet oxidation peaks for hydrogen are still observable when the tip was moving over the sample. Furthermore, it should be noticed the occurrence of non-zero current signals over the epoxy resin when the tip is moved from left to right towards the 
magnesium strip acting as the cathode, just following the application of the tip potential, indicating that some hydrogen bubbles may be pushed by the movement of the tip in this condition. Therefore, one must account for some interference of electrolyte convection occurring in the cell due to the evolution of several small hydrogen bubbles. On the other hand, hydrogen evolution from the corroding surface should originate increased $\mathrm{pH}$ values in the solution adjacent to the metal strip. However, black and blue lines given in Figure 5D for the anodically-behaving magnesium strip do not show any detectable $\mathrm{pH}$ gradient over the metal, i.e., neither alkalization nor acidification occurs in the nearby electrolyte. In this case, the recorded signal becomes very noisy, presumably due to hydrogen evolution, especially for the less aggressive condition at which hydrogen could be detected at the platinum UME (see black lines in Figures 5B and 5D). Heterogeneous $\mathrm{pH}$ distributions over the metal are only found under the most aggressive conditions attempted in this work (i.e., for the passage of $180 \mu \mathrm{A}$ total current), in which case some acidification occurs over the anodic strip accompanying very high rates of metal dissolution.

The spatial distribution of soluble magnesium species released from the corroding metal was also imaged by using Mg-ISME as the probe in potentiometric SECM operation. When a freely corroding magnesium strip was imaged, the metal dissolution rate was very small and could not be resolved. Accordingly, previous studies did not reveal measurable $\mathrm{Mg}^{2+}$ production in similar experiments [10], and for the unambiguous detection of a signal some other experimental approaches were required, such as the imaging of magnesium alloys [30] or the use of higher chloride concentrations [31]. Indeed, even the edge of the magnesium surface could not be easily distinguishable when scanning the sample in this case (not shown). In previous reports, enhanced chemical resolution with the SECM was attained following prior electrochemical activation of the metal surfaces by, for instance, the application of cathodic pulses to electrochemically reduce eventual corrosion products or passivating surface films. In this way, an enhancement of the substrate susceptibility towards corrosion was produced, and the response of the system was now measurable using electrochemical scanning techniques [41]. Thus, one magnesium surface was first cathodically-polarized by connecting the external circuit during 10 minutes, though no resistors were included this time in the circuit depicted in Figure 1.

After this polarization step was completed, the two magnesium surfaces were electrically disconnected, and several line scans were immediately recorded over the strip that acted as the cathode during the pre-polarization step. The scan rate was increased to $50 \mu \mathrm{m} \mathrm{s}^{-1}$ in order to obtain a reliable $2 \mathrm{D}$ image in the minimum possible recording time of the just pre-activated sample, taking advantage of the high stability of the solid-contact microelectrode assembly [31]. Figure 6 shows the weak electrochemical activation sensed over the magnesium strip, resulting in a reproducible and slightlyasymmetric peak amounting ca. $3.5 \times 10^{-6} \mathrm{~mol} \mathrm{dm}^{-3}$. Even without the pre-activation of the material, the degradation extent would not be expected to be significant, considering that hydrogen detection barely produced $45 \mathrm{pA}$ current increase, and hydroxide production resulted in $\mathrm{pH}$ around 11 (that is, hydroxide concentration in the $\mathrm{mM}$ range). Thereafter, it is no wonder that only a small detection of magnesium release was recorded over the freely corroding sample that was previously polarized as a cathode in the cell.

The effect of polarization between the two magnesium strips on the release of metal ions was further investigated in situ by recording $2 \mathrm{D}$ scans over the entire magnesium strips. The scan acquisition was also increased up to $50 \mu \mathrm{m} \mathrm{s}^{-1}$ in order to image the highly heterogeneous and dynamic activity of the whole metal surface. Some selected results are presented in Figure 7 for the magnesium strips, one acting as the cathode (left), and the other as the anode (right) in the electrochemical cell. The anodic conditions resulted in the unambiguous and spatially-distributed detection of magnesium ion release.

\section{Discussion}

By selecting an adequate combination of the operation modes available in SECM, the reaction leading to magnesium release and hydrogen evolution from separated magnesium cathodes and anodes was investigated. Magnesium ion selective microelectrodes (Mg-ISME's), antimony disc microelectrodes and platinum disc microelectrodes were employed for the spatially-resolved 
visualization of relevant chemical species participating in the corrosion reaction of magnesium, namely magnesium (II) ions, $\mathrm{pH}$ and hydrogen gas.

The development of a $\mathrm{pH}$ gradient around freely-corroding magnesium strips was shown in Figure 4B. Electrolyte alkalization is in good agreement with previous reports [10], and can be attributed to the low solubility of $\mathrm{Mg}(\mathrm{OH})_{2}$, with solubility constant $K_{s p}=3 \times 10^{-11}$ [11]. Furthermore, the shape of the $\mathrm{pH}$ gradient determined using the antimony disc microelectrode closely matches the amperometric current response recorded using a platinum disc microelectrode in a separate measurement (cf. Figure 4A). The latter signal is attributed to the oxidation of hydrogen gas evolving from the corroding surface. Both measurements were conducted on freshly prepared samples to prevent the corresponding maximum peaks to occur over the same point. Besides, no evidence of crevice corrosion was observed within the spatial resolution provided by these probes, indicating good joining between the insulating resin and the metallic strip. Unfortunately, these measurements do not allow the anodic and cathodic sites on the surface to be distinguished, neither to establish the origin of hydrogen evolution from the corroding magnesium surfaces.

Separation of the half-cell reactions was accomplished by imposing various degrees of polarization between two identical magnesium strips by means of the circuitry sketched in Figure 1. In this way, one of the surfaces was forced to be the anode in the resulting electrochemical cell, and the other magnesium strip became cathodically polarized. Figures 5-7 depict selected SECM measurements performed using this experimental arrangement. It was readily observed that the rate of the corrosion reaction was effectively modified by changing the values of the resistors employed in the external circuit.

In order to gain insights into the chemical and electrochemical processes participating in the system, the following half-cell reactions must be taken in account:

$$
\begin{aligned}
& \mathrm{Mg}^{0} \rightleftharpoons \mathrm{Mg}^{n+}+n e^{-} \\
& \mathrm{H}_{2} \mathrm{O}+2 e^{-} \rightleftharpoons 2 \mathrm{OH}^{-}+\uparrow \mathrm{H}_{2} \\
& \mathrm{O}_{2}+2 \mathrm{H}_{2} \mathrm{O}+4 e^{-} \rightleftharpoons 4 \mathrm{OH}^{-}
\end{aligned}
$$

Reactions (1) and (2) are well established as the main processes responsible for magnesium corrosion in neutral or alkaline solution at the open circuit potential, resulting in the production of metal cations and the evolution of hydrogen gas. In the framework of the on-going controversy regarding the occurrence of either mono or divalent nature for the oxidized magnesium species, $n$-valence magnesium ions have been introduced at this stage in the discussion, although only $\mathrm{Mg}^{2+}$ species can be detected with the Mg-ISME's employed in this work. Reaction (3) reflects the cathodic process which might take place if dissolved oxygen were to play the main role as oxidazing agent, unlikely to be the case under open circuit conditions [25], although we cannot discard that certain polarization conditions may partially allow this reaction to occur. In addition, the hydrolisis of the metal cations may subsequently happen (reaction 4). Finally, the frequently invoked magnesium (I) mechanism would require a redox reaction between $\mathrm{Mg}^{+}$species and water (reaction 5), and it is also included for discussion.

$$
\begin{aligned}
& \mathrm{Mg}^{n+}+x \mathrm{H}_{2} \mathrm{O} \rightleftharpoons \mathrm{Mg}(\mathrm{OH})_{x}^{(n-x)+}+x \mathrm{H}^{+} \\
& \mathrm{Mg}^{+}+2 \mathrm{H}_{2} \mathrm{O} \rightleftharpoons \mathrm{Mg}^{2+}+2 \mathrm{OH}^{-}+\uparrow H_{2}
\end{aligned}
$$

The scan lines shown in Figure 5 indicate that alkalization and vigorous hydrogen evolution occurred above the cathodically-polarized magnesium strip. This feature is moderate under mild polarization conditions, whereas strong effects observed under the most cathodic polarization. These features are consistent with the activity of the cathodic half-reaction during magnesium corrosion, corresponding to water reduction and hydrogen evolution (reaction 2) sketched in Figure 2. Therefore, the cathodic half-cell reaction produces greater $\mathrm{pH}$ gradients that actually affect the $\mathrm{pH}$ of adjacent solution and promote hydrogen evolution during magnesium corrosion. Since mild conditions result in similar signals as those measured in Figure 4 for the freely corroding magnesium, it can be 
concluded that the chemical changes related to cathodic activity effectively mask those related to the anodic sites when located in close proximity to each other, which is the case in a freely corroding magnesium substrate [7,9].

Weak evidences of hydrogen release were observed from the amperometric measurements across the anodically-polarized surface in Figure 5B, though hydrogen evolution was indeed readily observable through the videocamera. The HER occurs at a sufficient and homogeneous extent to unambiguosly produce some measurable signal only when the less aggressive anodic conditions were imposed to the system, and this feature is comparable to that found for the spontaneous situation shown in Figure 4A. The measured response diminishes upon imposing bigger anodic overvoltages (red and blue lines, Figure 5B). Conversely, the latter results in higher heterogeneity and noise levels, suggesting a more dynamic and/or localized evolution of hydrogen bubbles, whose motion probably provoke the observed noise. Thus, reaction (2) seems to occur on all of the anodic magnesium surfaces, although at a rather limited extent and in a highly heterogeneous fashion upon more aggressive conditions.

Similarly, no clear $\mathrm{pH}$ alterations occur for the systems where 35 and $110 \mu \mathrm{A}$ current was flowing, but the major activation using $180 \mu \mathrm{A}$ resulted in the clear acidification of the anodically-polarized metal (cf. Figure 5D). The lack of clear $\mathrm{pH}$ alteration in the two less aggressive conditions suggests that the limited occurrence of reaction (2), expected to result in local alkalization, is further compensated by local or general acidification at the anodes. Acidification around the anodic sites may be related to previous reports of local acidification attributed to pit formation on magnesium substrates [31]. In corrosion mechanisms, this feature is usually assigned to the hydrolysis of the hydrated ions, $\mathrm{Me}^{\mathrm{n}+}$, to form hydroxyl complexes according to the general equation:

$$
\mathrm{Me}^{n+}+x \mathrm{H}_{2} \mathrm{O} \rightleftharpoons \mathrm{Me}(\mathrm{OH})_{x}^{(n-x)+}+x \mathrm{H}^{+}
$$

The occurrence of this latter reaction and the extent of the subsequent local acidification is dependent on the acidity of the releasing metal ions. Since acidification caused by hydrolysis of $\mathrm{Me}^{2+}$ can be calculated from the stability constant of the $(\mathrm{Me}(\mathrm{OH}))^{(\mathrm{n}-1)^{+}}$complexes by assuming a concentration value for the metal ion [39], $\mathrm{pH}$ values were estimated as a function of the concentration of the dissolved metal. Unfortunately, these calculations indicate that $\mathrm{Mg}^{2+}$ might not be capable of producing major changes in the $\mathrm{pH}$ of a neutral corrosive environment, conversely to the strongly acidic distributions found in the case of iron dissolution [39].

In order to provide a satisfactory explanation for the reported acidification and the apparent heterogeneous weak release of hydrogen, it must be first noticed that impurities of nobler metals are present in the material under study, and they are known to increase the corrosion rate when present above a certain tolerance limit [42]. Considering the relatively high content of iron in the magnesium strip (0.05 wt. \%) and the strong acid character of the ferrous and ferric hydroxo-complexes, the contribution of such impurities towards $\mathrm{pH}$ modification, once oxidized during anodic polarization and/or microgalvanic corrosion, are likely to produce the observed acidification. Yet, further studies must be undertaken to unambiguously discern the origin of this acidification on anodically polarized magnesium surfaces. In the current experiments, since the anodic area covers the complete magnesium strip, the occurrence of local acidification is only observed under very vigorous metal dissolution conditions as to effectively counteract mixing with the bulk electrolyte.

In addition, it has been reported that the anodic degradation of impure magnesium leads to the development of a dark surface enriched in nobler metallic particles [11]. Those particles are expected to still behave cathodic with respect to the surrounding magnesium matrix when the whole material is anodically polarized. This would incur in the cathodic hydrogen evolution over these nobler particles as long as the microgalvanic coupling remains active. Such hydrogen evolution is certainly not as homogeneously distributed and active compared to the remaining magnesium surface behaving cathodically due to the imposed electric conditions. On the other hand, if the metal is further anodically polarized, even such impurities can be eventually dissolved and, due to the acidic nature of iron as main impurity, eventually contribute to the local acidification observed in the red curve shown in Figure 5D. 
The in situ consequences of the imposed electrical condition to the sample were also analyzed using the Mg-ISME as given in Figure 7 for the cathodically (left) and anodically (right) activated metal strips. In this way, the release of magnesium ions was effectively constrained to the anodicallypolarized metal sample, though originating a heterogeneous distribution of the metal species above the sample. It must be noticed that $\mathrm{Mg}$ (II) concentration values apparently greater than $0.1 \mathrm{~mol} \mathrm{dm}^{-3}$ cannot be considered to be quantitative because the calibration of the ISME was not linear beyond this higher $\left[\mathrm{Mg}^{2+}\right]$ limit. Yet, these results may indicate the occurrence of a vigorous degradation process for those experiments presenting overall current flows amounting 35 and $160 \mu \mathrm{A}$ (Figures 7D, E), depicting highly localized spots where determined magnesium exceeded this limit. This feature is evidenced by the rather positive potential value measured with the ISME, which can be regarded quantitative. Hence, it is reasonable to predict that magnesium concentration values over the linear range of the Mg-ISME's are attained at these hot spots dynamically developed over the surface behaving as the anode.

The greatest heterogeneities were observed under the most aggressive conditions presented here, corresponding to the passage of $160 \mu \mathrm{A}$ as the overall current (cf. Figure 7F). A justification for the prohibitively high values of magnesium concentration (up to $40 \mathrm{~mol} \mathrm{dm}^{-3}$ ), homogeneously found in a significant section of the surface, cannot be solely attributed to the vigorous dissolution process, as already discussed regarding Figures 7D and 7E. In contrast, it may be the result of the ionophore loosing selectivity as to respond also to proton activity whereas the environment becomes acidic [37]. In fact, simultaneous acidification of the electrolyte was clearly detected, as previously discussed for Figure 5D.

The dynamic character of the degradation process, involving passivation and local breakdown of the magnesium surfaces upon anodic polarization, is certainly influencing the given measurements. Indeed, the occurrence of such passivation-activation phenomena may explain the apparent contradictory observation of higher magnesium contents in Figure 7D compared to Figure 7E, the latter obtained under more aggressive conditions. Besides, another reason for this unexpected result might arise from the enhanced hydrogen bubbling, mainly observed in rather homogeneous fashion when the surface was less anodically polarized (that is, when $35 \mu \mathrm{A}$ global current was imposed), and occurring in a more heterogeneous way when the other anodic conditions were imposed. This may result in the unpredictable motion of the magnesium ions by electrolyte convection, in addition to diffusion. This possibility is supported by the previous observations of measurable hydrogen electro-oxidation along the black line in Figure 5B, together with the noisier signal depicted in Figure 5D, both recorded under the same conditions. An alternate explanation may arise from the precipitation of a magnesium(II) oxy-hydroxide layer with catalytic properties for the HER on the anodically-polarized metal [16], thus accounting for high rates of hydrogen evolution (imaged herein as finite oxidation currents at the $\mathrm{Pt}$ UME polarized at $0 \mathrm{~V}_{\mathrm{SCE}}$ ), with small concentration distributions of $\mathrm{Mg}^{2+}$ ions in the solution monitored with the Mg-ISME, even after polarization of the metal was stopped.

Regarding the visualization of the magnesium surfaces presenting a cathodic behaviour, the initial expectation was that featureless responses should be given by the Mg-ISME because such cathodic polarization conditions should simply protect the magnesium metal from corroding. In contrast, much lower (i.e., more negative) potential values were registered above the surfaces with cathodic behaviour, and thus translated into a low magnesium ion concentration through extrapolation of the calibration curve, as seen in the left side of Figure 7. Since, in principle, the Mg-ISME is not able to resolve a magnesium concentration below the micromolar range, further phenomena must be influencing the found potential values, and such estimated concentrations are certainly not real. Thus, experimental potential values have been added to the plot to avoid a misleading interpretation of the data. It must be noticed that the potential data may not correspond to those given in the calibration curve since the coordinate of origin differs from one electrode to another, which additionally originated a different scale range for the scans recorded over the anodically-polarized magnesium in Figure 7. These effects could be related to the imposed electric condition to the samples, which results in the establishment of an electric field. The stronger the electric field, the more cathodic were the potentials recorded using the liquid membrane electrode [31]. This correlates well with the reported ability of the ionophore cocktail to partially sense such electric field as well, resulting in a measurable signal response from the electrode, though in much lesser extent than for the eventually present $\mathrm{Mg}^{2+}$ species [31]. Thus, 
we expect that had some magnesium ions been produced from the cathodically active (that is, protected) surface, the Mg-ISME would probably have selectively detected them above the electric field signal. Further characterization of the Mg-ISME's must be conducted in the presence of both influencing parameters, magnesium cations and electric field, in order to unambiguously establish their eventual combined influence. In any case, the response of the Mg-ISME's over the anodicallypolarized surfaces should not be influenced by this fact [31]. As further influencing factors, up to now, no influence of the presence of hydrogen gas has been reported to affect the response or concentration of such ionophore cocktails, although at this stage it cannot be completely discarded either. Neither the local alkalization induced by the experimental procedure used in this work should cause any measurable effect [37].

\section{Conclusions}

The SECM has been presented as an essential technique to discern the corrosion mechanism of magnesium, mainly as the rare hydrogen evolution effect regards. With the assistance of a battery and a couple of resistances, the SECM has imaged distributions of hydrogen evolution, $\mathrm{pH}$ gradients and $\mathrm{Mg}^{2+}$ release over the separate magnesium anodes and cathodes, and results keep well correlation. No evidences of localized alkalization over the anodic Mg strip were found despite the increase of the corrosion current, but eventually local acidification instead. Such acidification was accompanied with major $\mathrm{Mg}^{2+}$ release as sensed by the Mg-ISME. The presence of impurities on the surface may also play a crucial role in the heterogeneous mechanism of magnesium corrosion, eventually promoting the hydrogen evolution and local acidification. In this way, new insights on the actual corrosion process of this material can be gathered using experimental strategies similar to those described here, thus contributing towards the elucidation of the actual mechanism of magnesium corrosion.

\section{Acknowledgements}

Financial support by the Spanish Ministry of Economy and Competitiveness (MINECO, Madrid) and the European Regional Development Fund (Brussels, Belgium), under grant CTQ2012-36787 is gratefully acknowledged. B.M.F.-P. thanks the University of La Laguna for a mobility grant to Pécs. The Hungarian Team wishes to thank the support of the "Environmental industry related innovative trans- and interdisciplinary research team development" in the University of Pécs knowledge base, the SROP-4.2.2.D-15/1/Konv-2015-0015 project.

\section{References}

[1] G. Song, Adv. Eng. Mater. 2005, 7, 563-586.

[2] N.T. Kirkland, N. Birbilis, M.P. Staiger, Acta Biomater. 2012, 8, 925-936.

[3] E. Ghali, W. Dietzel, K.-U. Kainer, J. Mater. Eng. Perform. 2013, 22, 2875-2891.

[4] J. Walker, S. Shadanbaz, T.B.F. Woodfield, M.P. Staiger, G.J. Dias, J. Biomed. Mater. Res. B 2014, 102, $1316-1331$.

[5] Z. Qiao, S. Shi, N. Hort, N.I.Z. Abiding, A. Atrens, Corros. Sci. 2012, 61, 185-207.

[6] A. Atrens, G.-L. Song, M. Liu, Z. Shi, F. Cao, S.S. Dargusch, Adv. Eng. Mater. 2015, 17, 400-453.

[7] G. Williams, H.N. McMurray, J. Electrochem. Soc. 2008, 155, C340-C349.

[8] J. Światowska, P. Volovitch, K. Ogle, Corros. Sci. 2010, 52, 2372-2378.

[9] N.T. Kirkland, G. Williams, N. Birbilis, Corros. Sci. 2012, 65, 5-9.

[10] J. Izquierdo, L. Nagy, I. Bitter, R.M. Souto, G. Nagy, Electrochim. Acta 2013, 87, 283-293.

[11] G. Williams, H. ap Ll. Dafydd, R. Grace, Electrochim. Acta 2013, 109, 489-501.

[12] S. Lebouil, A. Duboin, F. Monti, P. Tabeling, P. Volovitch, K. Ogle, Electrochim. Acta 2014, 124, 176-182. 
[13] G. Williams, N. Birbilis, H.N. McMurray, Electrochem. Commun. 2013, 36, 1-5.

[14] G. Williams, H. Dafydd, R. Subramanian, ECS Transactions 2013, 58, 23-34.

[15] M. Curioni, Electrochim. Acta 2014, 120, 284-292.

[16] N. Birbilis, A.D. King, S. Thomas, G.S. Frankel, J.R. Scully, Electrochim. Acta 2014, 132, $277-$ 283.

[17] M. Taheri, J.R. Kish, N. Birbilis, M. Danaie, E.A. McNally, J.R. McDermid, Electrochim. Acta 2014, 116, 396-403.

[18] A.C. Harms, S.N. Khanna, A.B. Chen, A.W. Castleman, J. Chem. Phys. 1994, 100, 3540-3544.

[19] R.I. Petty, A.W. Davidson, J. Kleinberg, J. Amer. Chem. Soc. 1954, 76, 363-366.

[20] A. Samaniego, B.L. Hurley, G.S. Frankel, J. Electroanal. Chem. 2015, 737, 123-128.

[21] G.S. Frankel, A. Samaniego, N. Birbilis, Evolution of hydrogen at dissolving magnesium surfaces, Corros. Sci. 2013, 70, 104-111.

[22] S. Thomas, N.V. Medhekar, G.S. Frankel, N. Birbilis, Curr. Opin. Solid State Mater. Sci. 2015, 19, 85-94.

[23] A.-M. Lafront, W. Zhang, S. Jin, R. Tremblay, D. Dubé, E. Ghali, Electrochim. Acta 2005, 51, 489-501.

[24] O.V. Karavai, A.C. Bastos, M.L. Zheludkevich, M.G. Taryba, S.V. Lamaka, M.G.S. Ferreira, Electrochim. Acta 2010, 55, 5401-5406.

[25] S. Thomas, J. Izquierdo, N. Birbilis, R.M. Souto, Corrosion 2015, 71, 171-183.

[26] K. Fushimi, K.A. Lill, H. Habazaki, Electrochim. Acta 2007, 52, 4246-4253.

[27] S.S. Jamali, S.E. Moulton, D.E. Tallman, M. Forsyth, J. Weber, G.G. Wallace, Corros. Sci. 2014, 86, 93-100.

[28] X. Liu, T. Zhang, Y. Shao, G. Meng, F. Wang, Corros. Sci. 2009, 51, 1772-1779.

[29] W. Liu, F. Cao, Y. Xia, L. Chang, J. Zhang, Electrochim. Acta 2014, 134, 377-388.

[30] R.M. Souto, A. Kiss, J. Izquierdo, L. Nagy, I. Bitter, G. Nagy, Electrochem. Commun. 2013, 26, 25-28.

[31] J. Izquierdo, A. Kiss, J.J. Santana, L. Nagy, I. Bitter, H.S. Isaacs, G. Nagy, R.M. Souto, J. Electrochem. Soc. 2013, 160, C451-C459.

[32] U.M. Tefashe, M.E. Snowden, P.D. Ducharme, M. Danaie, G.A. Botton, J. Mauzeroll, J. Electroanal. Chem. 2014, 720-721, 121-127.

[33] S. Bender, J. Goellner, A. Heyn, S. Schmigalla, Mater. Corros. 2012, 63, 707-712.

[34] M. Etienne, A. Schulte, W. Schuhmann, Electrochem. Commun. 2004, 6, 288-293.

[35] C. Kranz, M. Ludwig, H.E. Gaub, W. Schuhmann, Adv. Mater. 1995, 7, 568-571.

[36] J. Izquierdo, L. Nagy, Á. Varga, J.J. Santana, G. Nagy, R.M. Souto, Electrochim. Acta 2011, 56, 8846-8850.

[37] K. Tóth, E. Lindner, M. Horváth, J. Jeney, E. Pungor, I. Bitter, B. Ágai, L. Töke, Electroanal. 1993, 5, 781-790.

[38] R.M. Souto, L. Fernández-Mérida, S. González, Electroanal. 2009, 21, 2640-2646.

[39] S.V. Lamaka, R.M. Souto, M.G.S. Ferreira in Microscopy: Science, Technology, Applications and Education, Vol. 3 (Eds.: A. Méndez-Vilas, J. Díaz), Formatex Research Center, Badajoz, Spain, 2010, pp. 2162-2173.

[40] R.M. Souto, J. Izquierdo, J.J. Santana, A. Kiss, L. Nagy, G. Nagy in Current Microscopy Contributions to Advances in Science and Technology, Vol. 2 (Ed.: A. Méndez-Vilas), Formatex Research Center, Badajoz, Spain, 2012, pp. 1407-1415.

[41] J. Izquierdo, L. Martín-Ruíz, B.M. Fernández-Pérez, R. Rodríguez-Raposo, J.J. Santana, R.M. Souto, J. Electroanal. Chem. 2014, 728, 148-157. 
[42] M. Liu, P.J. Uggowitzer, A.V. Nagasekhar, P. Schmutz, M. Easton, G.-L. Song, A. Atrens, Corros. Sci. 2009, 51, 602-619.

Table 1. Composition of the mixture employed to produce the cocktail for the $\mathrm{Mg}^{2+}$ ion selective microelectrodes.

\begin{tabular}{|l|l|l|}
\hline \multirow{2}{*}{ Component } & \multicolumn{2}{|c|}{ Quantities for $200 \mu \mathrm{L}$ of the mixture } \\
\cline { 2 - 3 } & \multicolumn{1}{|c|}{ Content } & wt.\% \\
\hline Tetrahydrofurane (THF) & $100 \mu \mathrm{L}$ & \\
\hline Poly(vinyl chloride) (PVC) & $7.68 \mathrm{mg}$ & 5.06 \\
\hline $\begin{array}{l}\text { bis-N,N-dicyclohexyl- } \\
\text { malonamide }\end{array}$ & $2.23 \mathrm{mg}$ & 1.47 \\
\hline $\begin{array}{l}\text { Potassium tetrakis(4- } \\
\text { chlorophenyl)borate } \\
\text { (PTCB) }\end{array}$ & $2.13 \mathrm{mg}$ & 1.40 \\
\hline $\begin{array}{l}\text { ortho-nitrophenyl octyl } \\
\text { ether (o-NPOE) }\end{array}$ & $139.79 \mathrm{mg}$ & 92.07 \\
\hline
\end{tabular}

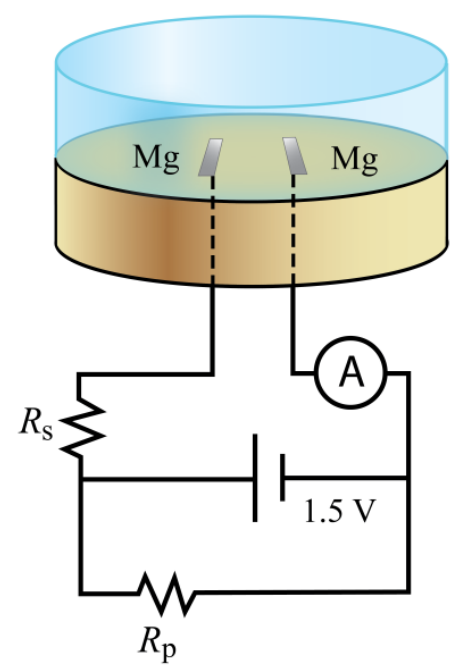

Fig. 1. Scheme of the circuitry employed to control the electrical state of the magnesium strips in the sample. 
A

SG-TC amperometric operation

(detection of $\mathrm{H}_{2}$ evolution)

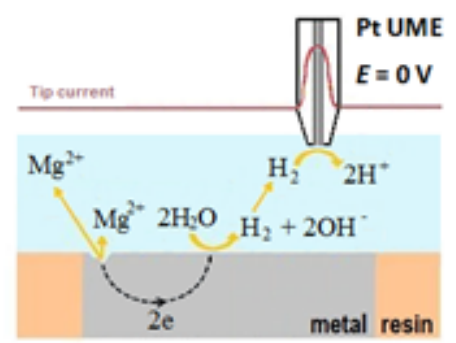

B

Potentiometric operation

(measurement of $\mathrm{pH}$ )

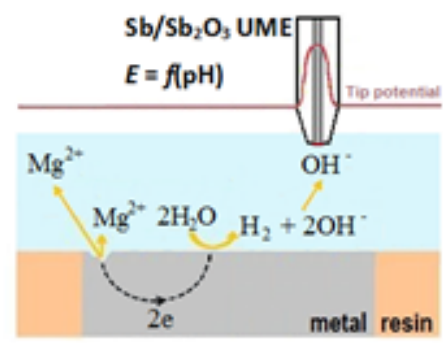

C

Potentiometric operation (measurement of $\mathrm{Mg}^{2+}$ concentration)

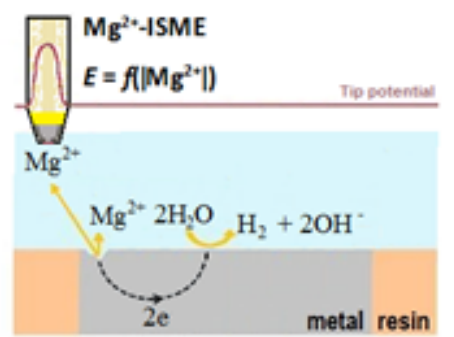

D

Negative feedback amperometric operation

(reduction of soluble $\mathrm{O}_{2}$ )

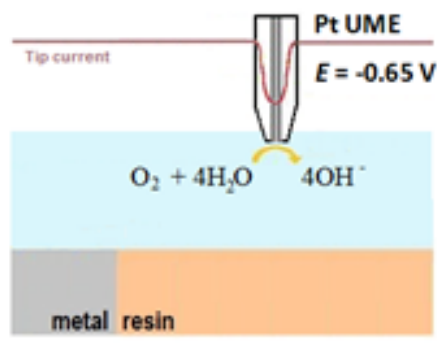

E

Alternating current operation

(local conductivity)

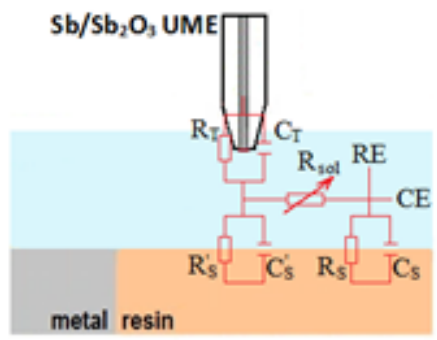

Fig. 2. The different modes of SECM operation employed in this work: A) Amperometric Sample Generation-Tip Collection for the detection of hydrogen evolution using a Pt UME; B) Potentiometric measurement of $\mathrm{pH}$ using a $\mathrm{Sb} / \mathrm{Sb}_{2} \mathrm{O}_{3} \mathrm{UME}$; C) Potentiometric measurement of magnesium ion concentration using a Mg(II)-ISME; D) Amperometric negative feedback operation of a Pt UME for oxygen reduction over the resin (used for tip positioning); and E) Alternating-current operation employed for tip positioning of an ion selective probe.
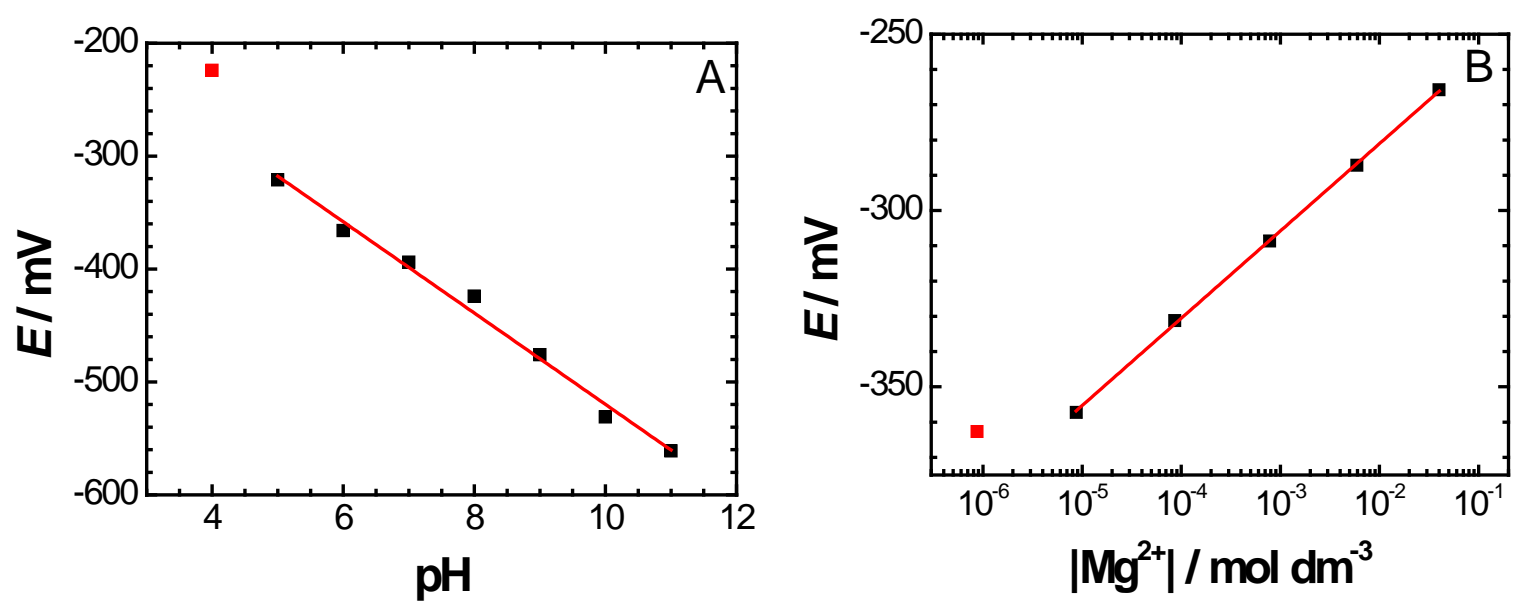

Fig. 3. Calibration of the (A) antimony-based and (B) magnesium-ion selective microelectrodes as a function of the activity of hydrogen and magnesium ions, respectively. Red dots in the plots were not considered for the establishment of the corresponding linear correlations. 

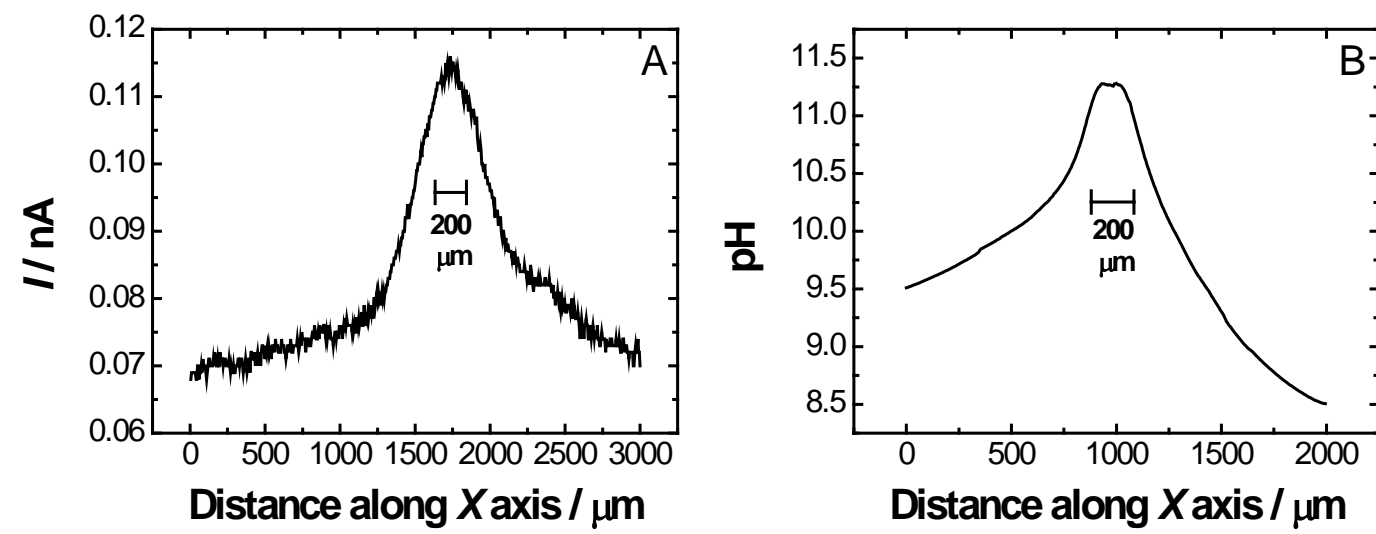

Distance along $X$ axis $/ \mu \mathrm{m}$

Fig. 4. SECM scan lines recorded over a freely corroding magnesium strip (i.e., no polarization applied to the metal). (A) Amperometric response of the Pt-UME for the detection of hydrogen evolution from the corroding metal; and (B) $\mathrm{pH}$ distribution recorded using the $\mathrm{Sb} / \mathrm{Sb}_{2} \mathrm{O}_{3}$ electrode. Scan rate: $10 \mu \mathrm{m} \mathrm{s}^{-1}$. The $200 \mu \mathrm{m}$ scale bar indicates the approximate location of the magnesium strip.

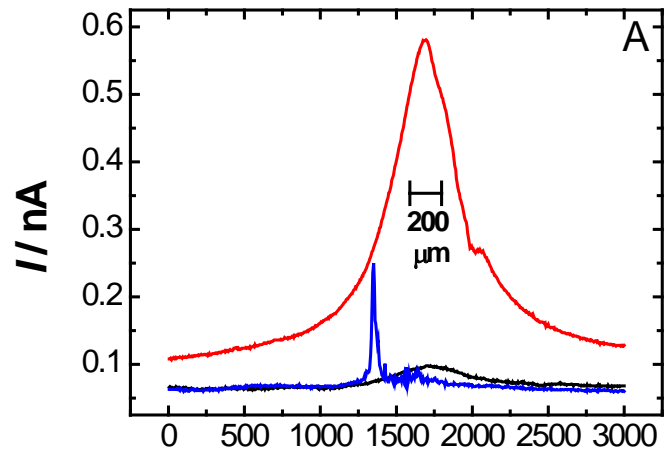

Distance along $X$ axis $/ \mu \mathrm{m}$

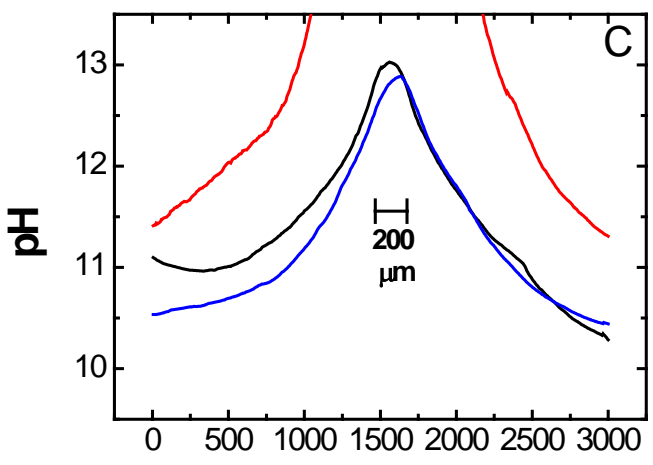

Distance along $X$ axis $/ \mu \mathrm{m}$
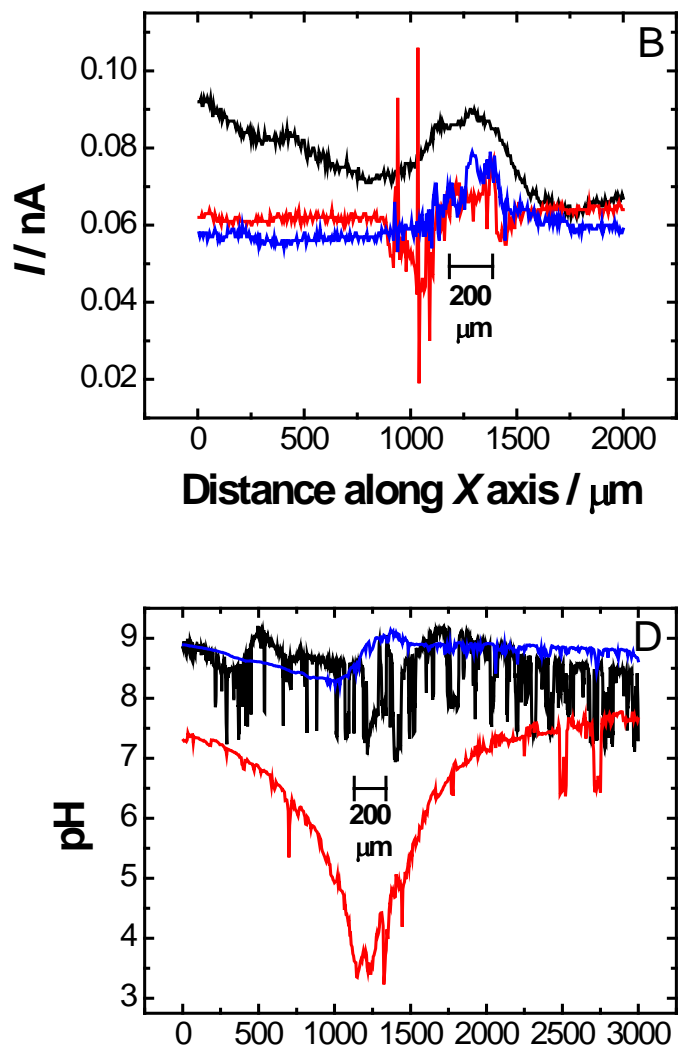

Distance along $X$ axis $/ \mu \mathrm{m}$

Fig. 5. SECM scan lines recorded over the polarized magnesium strips: $(A, C)$ the strip subjected to cathodic polarization, and (B,D) the strip subjected to anodic polarization. SECM operation modes: $(A, B)$ Amperometric response of the Pt-UME for the detection of hydrogen bubbles formation; and (C,D) $\mathrm{pH}$ distribution gradients recorded with the antimony electrode over the polarized magnesium strips under polarization. Total current flowing between the polarized magnesium strips: (black) 35, (blue) 110 , and (red) $180 \mu \mathrm{A}$. Scan rate: $10 \mu \mathrm{m} \mathrm{s}^{-1}$. The $200 \mu \mathrm{m}$ scale bar indicates the approximate location of the magnesium strip. 


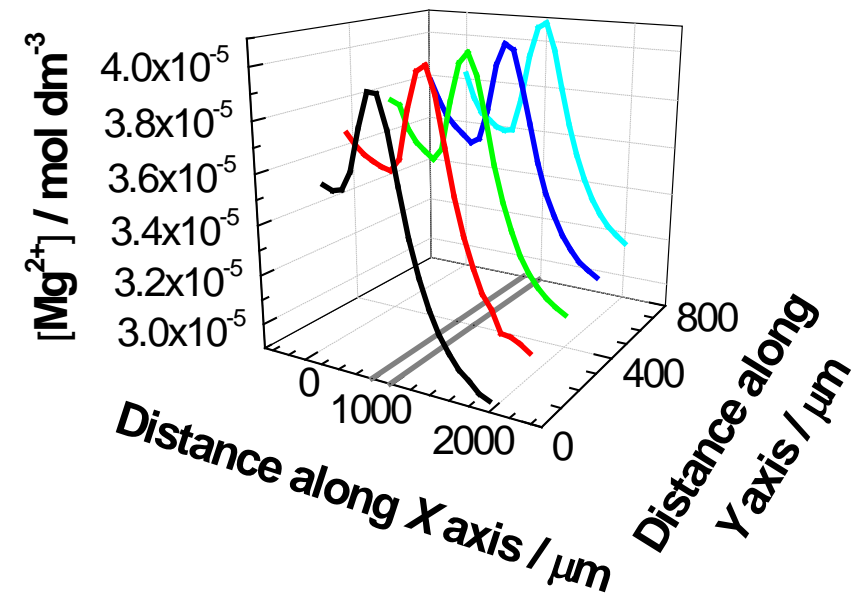

Fig. 6. SECM scan lines recorded with the Mg-ISME over the freely corroding magnesium sample following a $10 \mathrm{~min}$ polarization step as cathode. Scan rate: $50 \mu \mathrm{m} \mathrm{s}^{-1}$. Grey lines in the $X Y$ plane indicate the approximate location of the Mg strip.
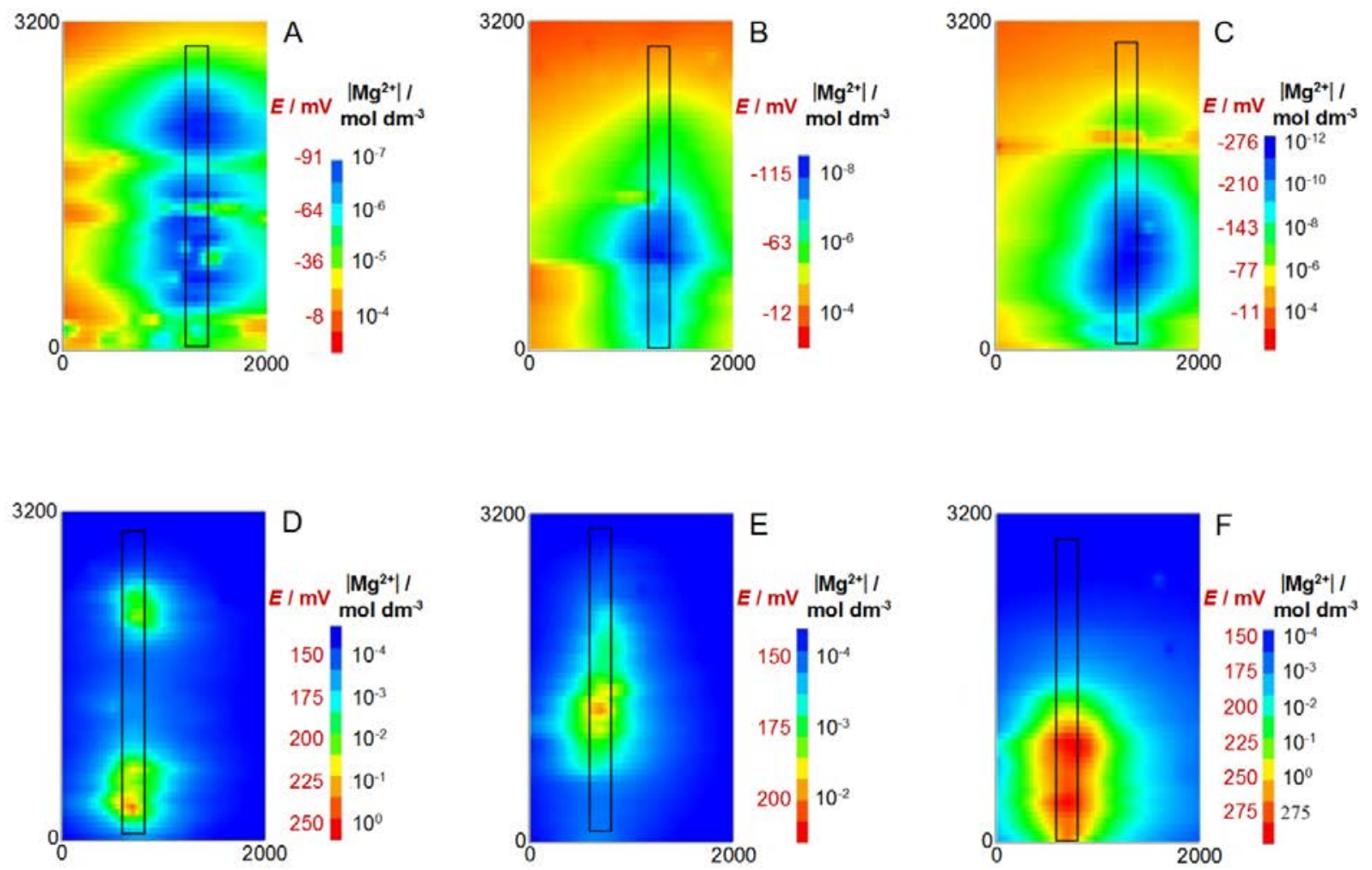

Fig. 7. SECM scans recorded with the Mg-ISME over the polarized magnesium strips: $(A, B, C)$ the strip subjected to cathodic polarization, and (D,E,F) the strip subjected to anodic polarization. The images show the concentration distribution of $\mathrm{Mg}$ (II) ions released from the corroding magnesium strips as result of external polarization. The potential values actually measured are also given. Total current flowing between the magnesium strips during polarization: (A,D) 35, (B,E) 110, and (C,F) 160 $\mu \mathrm{A}$. Scan rate: $50 \mu \mathrm{m} \mathrm{s}^{-1}$. The rectangles drawn in the figure indicate the approximate location of the magnesium strips. 